\title{
Spin-Dependent Transport through a Single-Wall Carbon Nanotube Quantum Dot with an $S=1$ Molecule
}

\author{
A. PŁomińska And I. Weymann \\ Faculty of Physics, Adam Mickiewicz University, Umultowska 85, 61-614 Poznań, Poland
}

\begin{abstract}
Transport properties of an $S=1$ molecule attached to a single-wall carbon nanotube quantum dot, which is coupled to two external ferromagnetic leads, are analyzed in the sequential tunneling regime. The magnetizations of the leads are assumed to form either a parallel or an antiparallel magnetic configuration. The calculations are performed by using the real-time diagrammatic technique in the lowest order perturbation theory with respect to the tunnel coupling. It is shown that the presence of the molecule strongly affects the bias voltage dependence of the current and differential conductance in both magnetic configurations, as well as the resulting tunnel magnetoresistance. Negative (greatly enhanced) tunnel magnetoresistance is found in the case of antiferromagnetic (ferromagnetic) coupling between the nanotube and molecule.
\end{abstract}

DOI: $10.12693 /$ APhysPolA.127.475

PACS: 72.25.-b, 75.50.X.x, 85.75.-d

\section{Introduction}

Transport properties of various magnetic molecules have been a subject of intensive studies for several years [1]. When attached to external ferromagnetic leads, the molecules can exhibit a considerable tunnel magnetoresistance (TMR) effect [2]. Such nanostructures are thus very interesting for molecular spintronics and potentially important for future applications in information storage technologies [3]. In this paper we study spinresolved transport properties of single-wall carbon nanotube quantum dot, with attached magnetic molecule of spin $S=1$, weakly coupled to external ferromagnetic leads. The calculations are performed by using the realtime diagrammatic technique in the lowest-order perturbation theory with respect to the coupling to external leads. We show that, depending on the type of exchange interaction between the molecule and nanotube, the TMR can be either greatly enhanced or can become negative.

\section{Model and method}

The considered system consists of a single-wall carbon nanotube quantum dot, with an exchange coupled spin $S=1$ molecule, which is tunnel-coupled to the left $(r=L)$ and right $(r=R)$ ferromagnetic leads, see Fig. 1 . The magnetizations of the leads are assumed to form either parallel or antiparallel magnetic configuration. The coupling between the molecule and the nanotube is described by $J_{S}$ and can be either ferromagnetic $\left(J_{S}>0\right)$ or antiferromagnetic $\left(J_{S}<0\right)$. The molecule is characterized by an uniaxial magnetic anisotropy $D$ and its easy axis coincides with the direction of magnetizations of the leads. For the nanotube we assume that only two levels, each belonging to different subband, take part in

corresponding author; e-mail: ania1990ftr@wp.pl transport. Consequently, the Hamiltonian of the system can be written as, $H=H_{e l}+H_{m o l}+H_{t u n}$, where

$$
H_{e l}=\sum_{r k \sigma} \varepsilon_{r k \sigma} c_{r k \sigma}^{\dagger} c_{r k \sigma}
$$

describes the noninteracting electrons in the leads, with $c_{r k \sigma}^{\dagger}$ creating a spin- $\sigma$ electron with momentum $k$ in lead $r$ of energy $\varepsilon_{r k \sigma}$. The second term of the Hamiltonian describes the carbon nanotube quantum dot with coupled molecule of spin $S$. It acquires the form $[2,4]$

$$
\begin{gathered}
H_{m o l}=\sum_{j \sigma} \varepsilon_{j} n_{j \sigma}+\frac{U}{2} N^{2}+J N_{\uparrow} N_{\downarrow}-J_{S} \boldsymbol{S} \cdot \boldsymbol{s} \\
-D S_{z}^{2},
\end{gathered}
$$

where $n_{j \sigma}=d_{j \sigma}^{\dagger} d_{j \sigma}$ is the particle number operator for an electron of spin $\sigma$ in the $j$ th $(j=1,2)$ level of the nanotube, $\varepsilon_{j}$ denotes the respective energy, with $\varepsilon_{1}=\varepsilon$, $\varepsilon_{2}=\varepsilon+\delta$, where $\delta$ is the energy mismatch between the subbands of the nanotube. The charging energy of the nanotube is denoted by $U$, while $J$ describes the exchange interaction, with $N=N_{\uparrow}+N_{\downarrow}$ and $N_{\sigma}=\sum_{j} n_{j \sigma}$. The molecule is modeled by the giant spin Hamiltonian where



Fig. 1. Schematic of a carbon nanotube quantum dot with spin $S=1$ molecule coupled to external ferromagnetic leads. The nanotube is assumed to have two levels of energy $\varepsilon$ and $\varepsilon+\delta . \Gamma_{L}^{\sigma}\left(\Gamma_{R}^{\sigma}\right)$ denotes the spindependent coupling to ferromagnetic left (right) lead and $J_{S}$ is the exchange coupling between the molecule and nanotube. 
$\boldsymbol{S}$ is the corresponding spin operator and $S_{z}$ its $z$ th component. $J_{S}$ denotes the exchange coupling between the electrons occupying the nanotube and the molecule, with $s=\frac{1}{2} \sum_{j \sigma \sigma^{\prime}} d_{j \sigma}^{\dagger} \boldsymbol{\sigma}_{\sigma \sigma^{\prime}} d_{j \sigma^{\prime}}$ being the spin operator of electrons in the nanotube and $\boldsymbol{\sigma}$ is the Pauli spin operator. The coupling between the nanotube and the leads is described by the tunneling Hamiltonian

$$
H_{t u n}=\sum_{r=L, R} \sum_{j=1,2} t_{r j} \sum_{k \sigma}\left(c_{r k \sigma}^{\dagger} d_{j \sigma}+d_{j \sigma}^{\dagger} c_{r k \sigma}\right),
$$

where $t_{r j}$ denotes the tunnel matrix elements between the $r$ th lead and the $j$ th level of the nanotube. The respective strength of the coupling between lead $r$ and level $j$ is given by $\Gamma_{r j}^{\sigma}=2 \pi \rho_{r}^{\sigma} t_{r j}^{2}$, with $\rho_{r}^{\sigma}$ denoting the spin-dependent density of states of lead $r$. It can be then expressed as $\Gamma_{r j}^{\sigma}=\left(1 \pm p_{r}\right) \Gamma_{r j}$, where $p_{r}$ is the spin polarization of lead $r$ and $\Gamma_{r j}=\left(\Gamma_{r j}^{\uparrow}+\Gamma_{r j}^{\downarrow}\right) / 2$. In our considerations we assume that the system is fully symmetric, i.e. $p_{r} \equiv p$ and $\Gamma_{r j} \equiv \Gamma$.

To calculate the transport properties of the system we employ the real-time diagrammatic technique $[5,6]$. Since we consider the weak coupling regime and focus in our analysis on sequential processes, only the lowest order of perturbative expansion of the reduced density matrix of the system and operators of interest is needed. The occupation probabilities $P_{\chi}$ of many-body states $|\chi\rangle$ of the system, such that $H_{m o l}|\chi\rangle=\varepsilon_{\chi}|\chi\rangle$, can be found from [6]

$$
(\boldsymbol{W} \boldsymbol{P})_{\chi}=\Gamma \delta_{\chi \chi_{0}}
$$

where $\boldsymbol{W}$ and $\boldsymbol{P}$ denote the self-energy matrix and probability vector, respectively. The elements of $\boldsymbol{W}$ are given by $W_{\chi \chi^{\prime}}=W_{\chi \chi^{\prime}}^{L}+W_{\chi \chi^{\prime}}^{R}$, where

$$
\begin{aligned}
& W_{\chi \chi^{\prime}}^{r}=2 \pi \sum_{\sigma} \rho_{r}^{\sigma}\left\{f_{r}\left(\varepsilon_{\chi}-\varepsilon_{\chi^{\prime}}\right)\left|\sum_{j} t_{r j}\left\langle\chi\left|d_{j \sigma}^{\dagger}\right| \chi^{\prime}\right\rangle\right|^{2}\right. \\
& \left.\quad+\left[1-f_{r}\left(\varepsilon_{\chi^{\prime}}-\varepsilon_{\chi}\right)\right]\left|\sum_{j} t_{r j}\left\langle\chi\left|d_{j \sigma}\right| \chi^{\prime}\right\rangle\right|^{2}\right\}
\end{aligned}
$$

for $\chi \neq \chi^{\prime}$ and $W_{\chi \chi}^{r}=-\sum_{\chi^{\prime} \neq \chi} W_{\chi^{\prime} \chi}^{r}$, with $f_{r}(\varepsilon)=$ $1 /\left[\mathrm{e}^{\left(\varepsilon-\mu_{r}\right) / T}+1\right]$ and $\mu_{r}$ being the electrochemical potential of lead $r$. On the other hand, the current flowing through the system can be found from [6]

$$
I=\frac{e}{2 \hbar} \operatorname{Tr}\left\{\boldsymbol{W}^{I} \boldsymbol{p}\right\},
$$

where the elements of self-energy matrix $\boldsymbol{W}^{I}$ are given by, $W_{\chi \chi^{\prime}}^{I}=\left[\Theta\left(N_{\chi^{\prime}}-N_{\chi}\right)-\Theta\left(N_{\chi}-N_{\chi^{\prime}}\right)\right]\left(W_{\chi \chi^{\prime}}^{R}-W_{\chi \chi^{\prime}}^{L}\right)$, with $N_{\chi}$ the electron number of state $|\chi\rangle$ and $\Theta(x)$ the step function.

\section{Numerical results and discussion}

In the following, we present and discuss the numerical results on the current and differential conductance of the system in the parallel (P) and antiparallel (AP) magnetic configuration. We also study the bias dependence of the tunnel magnetoresistance, which is defined as, TMR = $I^{P} / I^{A P}-1$, where $I^{P}\left(I^{A P}\right)$ is the current flowing in the parallel (antiparallel) configuration of the system.

The occupancy of the nanotube can be changed by sweeping the gate voltage. In the linear response regime,

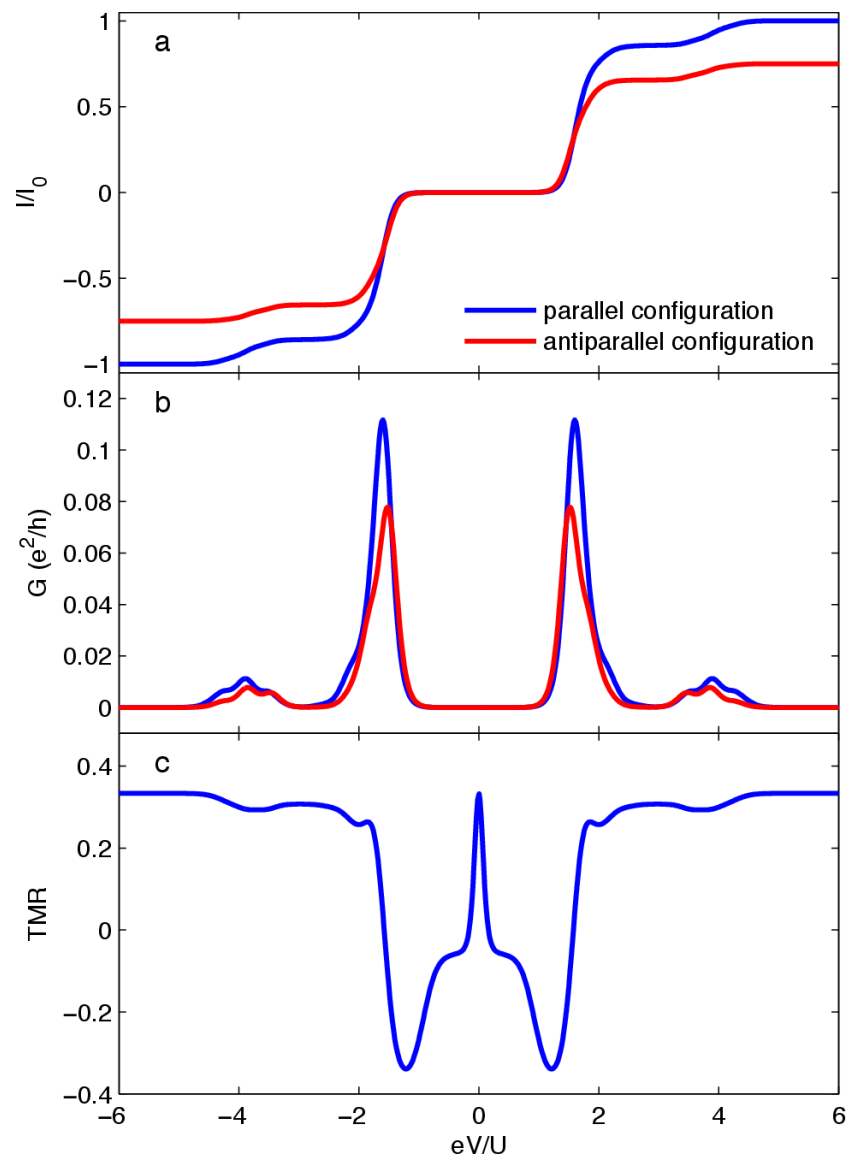

Fig. 2. The bias voltage dependence of (a) the current and (b) the differential conductance in the parallel and antiparallel magnetic configurations as well as (c) the resulting TMR in the case of antiferromagnetic coupling between the molecule and nanotube, $J_{S}<0$. The parameters are: $\varepsilon / U=-2.5, J / U=0.4, \delta / U=0$, $J_{S} / U=-0.15, D / U=0.1, T / U=0.05, \Gamma / U=0.01$, with $U \equiv 1$ the energy unit and $p=0.5$. The current is expressed in units of $I_{0}=e \Gamma / \hbar$.

each time the energy level of the nanotube crosses the Fermi energy of the leads, there is a resonant peak in the linear conductance and the occupation changes by one. However, the sequence of ground states depends considerably on the internal parameters of the nanotube. When the energy mismatch between the subbands $\delta$ is larger than the exchange interaction $J$ of the nanotube, $\delta>J$, with changing the gate voltage, the first level becomes first fully occupied and then the second level starts being populated, so that the doubly occupied state of the nanotube is a singlet. Nevertheless, in the opposite case, $\delta<J$, in the doubly occupied state the electrons occupy different levels of the nanotube and form a triplet. Here we focus on the latter situation, $\delta<J$, as more interesting as far as the transport properties in the case of additional molecule of spin $S=1$ are considered. More specifically, we study the spin-resolved transport properties in the nonlinear response regime for gate voltages such that the nanotube is doubly occupied at equilibrium. 




Fig. 3. The same as in Fig. 2 calculated in the case of ferromagnetic coupling between the molecule and nanotube, $J_{S} / U=0.15$.

The bias voltage dependence of the current and the differential conductance in both magnetic configurations as well as the resulting TMR in the case of antiferromagnetic coupling between the molecule and nanotube $\left(J_{S}<0\right)$ is shown in Fig. 2. At low bias voltage the system is in the Coulomb blockade regime and the current starts flowing when the voltage reaches a threshold voltage. With increasing bias voltage, both $I^{\mathrm{P}}$ and $I^{\mathrm{AP}}$ exhibit typical Coulomb staircase characteristics, see Fig. 2a. This is reflected in the bias dependence of the differential conductance shown in Fig. 2b, which displays typical peaks characteristic of single-electron charging effects. The difference between the two magnetic configurations of the system is reflected in the TMR. At zero bias, the TMR takes the value [7], TMR $=p^{2} /\left(1-p^{2}\right)$, which for assumed spin polarization of the leads gives, $\mathrm{TMR}=1 / 3$. Then, with increasing the bias voltage, TMR suddenly drops and becomes negative just before the threshold voltage, to increase again when the rate of sequential processes increases.
The transport properties can be changed in the case when the exchange coupling between the molecule and nanotube is of ferromagnetic type, see Fig. 3. Although the current exhibits typical Coulomb staircase dependence on the bias voltage and differential conductance has characteristic peaks, the behavior of the TMR is now completely different. For $J_{S}>0$, transport takes part through high-spin many-body states of the system. In the antiparallel configuration this may lead to high nonequilibrium spin accumulation, which increases the difference between the currents $I^{\mathrm{AP}}$ and $I^{\mathrm{P}}$. This leads to large positive TMR, which for voltages just before the threshold takes values much larger than those predicted for single-wall carbon nanotube quantum dots in the absence of additional molecule [8].

In summary, we have studied the spin-resolved transport properties of single-wall carbon nanotube quantum dots coupled to external ferromagnetic leads with an attached molecule of spin $S=1$. We have shown that the presence of additional molecule of high spin can greatly affect the magnetoresistive properties of the system. In the case of antiferromagnetic (ferromagnetic) exchange coupling between the molecule and nanotube we have found negative (considerably enhanced) tunnel magnetoresistance for voltages around the threshold voltage.

\section{Acknowledgments}

This work was supported by the National Science Center in Poland as the Project No. DEC2013/10/E/ST3/00213.

\section{References}

[1] D. Gatteschi, R. Sessoli, and J. Villain, Molecular Nanomagnets, Oxford University Press, New York 2006.

[2] M. Misiorny, I. Weymann, J. Barnaś, Phys. Rev. B 79, 224420 (2009).

[3] L. Bogani, W. Wernsdorfer, Nature Mat. 7, 179 (2008).

[4] Y. Oreg, K. Byczuk, B.I. Halperin, Phys. Rev. Lett. 85, 365 (2000).

[5] H. Schoeller, G. Schön, Phys. Rev. B 50, 18436 (1994).

[6] A. Thielmann, M.H. Hettler, J. König, and G. Schön, Phys. Rev. Lett. 95, 146806 (2005).

[7] I. Weymann, J. König, J. Martinek, J. Barnaś, and G. Schön, Phys. Rev. B 72, 115334 (2005).

[8] I. Weymann, J. Barnaś and S. Krompiewski, Phys. Rev. B 76, 155408 (2007). 